\title{
GCU
}

Glasgow Caledonian

University

University for the Common Good

\section{Perspectives on pre-exposure prophylaxis for people who inject drugs in the context of an HIV outbreak: a qualitative study}

Smith, Matt; Elliott, Lawrie; Hutchinson, Sharon J.; Metcalfe, Rebecca; Flowers, Paul; McAuley, Andrew

Published in:

International Journal of Drug Policy

DOI:

10.1016/j.drugpo.2020.103033

Publication date:

2021

Document Version

Author accepted manuscript

Link to publication in ResearchOnline

Citation for published version (Harvard):

Smith, M, Elliott, L, Hutchinson, SJ, Metcalfe, R, Flowers, P \& McAuley, A 2021, 'Perspectives on pre-exposure prophylaxis for people who inject drugs in the context of an HIV outbreak: a qualitative study', International Journal of Drug Policy, vol. 88, 103033. https://doi.org/10.1016/j.drugpo.2020.103033

\section{General rights}

Copyright and moral rights for the publications made accessible in the public portal are retained by the authors and/or other copyright owners and it is a condition of accessing publications that users recognise and abide by the legal requirements associated with these rights.

Take down policy

If you believe that this document breaches copyright please view our takedown policy at https://edshare.gcu.ac.uk/id/eprint/5179 for details of how to contact us. 
Title: Perspectives on Pre-exposure Prophylaxis for People Who Inject Drugs in the context of an HIV outbreak: a qualitative study.

Authors: Matt Smith ${ }^{a}$, Lawrie Elliott ${ }^{a}$, Sharon J. Hutchinson ${ }^{a, b}$, Rebecca Metcalfe ${ }^{a, c}$,

Paul Flowers ${ }^{\mathrm{d}}$, Andrew McAuleya,b

Affiliations: a) School of Health and Life Sciences, Glasgow Caledonian University, Cowcaddens Road, Glasgow, UK G4 OBA b) Health Protection Scotland, Meridian Court, Glasgow, UK, G4 OBA c) NHS Greater Glasgow and Clyde, Sandyford Sexual Health Service, Glasgow, UK G3 7NB d). School of Psychological Sciences \& Health Room, Graham Hills Building, 40 George Street, Glasgow, G1 1QE

Matt.smith@gcu.ac.uk

Lawrie.elliott@gcu.ac.uk

Sharon.hutchinson2@nhs.net

rebeccametcalfe@nhs.net

Paul.flowers@strath.ac.uk

Andrew.mcauley@nhs.net

Corresponding author: Matt Smith

Present address: School of Health and Life Sciences, Glasgow Caledonian

University, Cowcaddens Road, Glasgow, G4 OBA

Declarations of interest:

Matt Smith: None

Lawrie Elliott: has received funding from NHS Greater Glasgow and Clyde during this study. 
Sharon Hutchinson: has received honoraria from Gilead, unrelated to submitted work. Has received funding from NHS Greater and Glasgow and Clyde and Health Protection Scotland during this study.

Rebecca Metcalfe: None

Paul Flowers: Currently a trustee of the National AIDS Trust. Has received grants from ViiV and Gilead, unrelated to submitted work.

Andrew McAuley: None

Funding: This study was funded by the NHS Greater Glasgow and Clyde Health Board. 
Perspectives on Pre-exposure Prophylaxis for People Who Inject Drugs in the context of an HIV outbreak: a qualitative study.

Matt Smith, Lawrie Elliott, Sharon Hutchinson, Rebecca Metcalfe, Paul Flowers, Andrew McAuley

Keywords: PrEP, HIV, Injecting Drug Use, Outbreak, PWID

\section{Abstract:}

Background: There is an ongoing HIV outbreak among people who inject drugs (PWID) in Glasgow, Scotland, and one response which has not yet been widely implemented is the provision of Pre-exposure prophylaxis (PrEP). PrEP is the use of HIV anti-retrovirals prior to HIV infection to provide a barrier to infection. This has been shown to be effective among various at-risk populations in preventing HIV spread. The present study aimed to explore views of PWID who might benefit from PrEP provision and Service Providers working with PWID to understand will to use PrEP and literacy of PrEP, contributing to the development of a PrEP service.

Methods: A qualitative approach was taken, with semi structured interviews conducted in Glasgow at two third sector service sites. 11 Service Providers and 21 PWID participated in the study. Data was analysed thematically.

Results: Participants, both PWID and Service Providers, were keen to engage with PrEP and perceived substantial potential benefits of PrEP for this population. Potential barriers to engagement were identified as a lack of health literacy, motivation, and self-ascribed risk, as well as the overwhelming unpredictability of substance use. Participants wanted PrEP to be provided within already existing 
structures, particularly community pharmacies, and for promotion and provision to involve peers.

Conclusion: This sample reported willingness to engage with PrEP, and suggested there is a specific need among PWID for PrEP. . However, PWID have specific lived experienced contexts and needs, and are burdened by social and economic marginalisation and inequality at every level. This contrasts them from other populations currently being provided with PrEP, and must be considered in the development of provision. 


\section{Background}

People who inject drugs (PWID) are at increased risk of HIV transmission (Hope et al., 2014). Risk factors emerge from both injecting behaviours (sharing of needles, syringes and associated paraphernalia) and sexual transmission. PWID are 22 times more at risk of acquiring HIV than the general population and of an estimated 11.8 million PWID worldwide, around $13.1 \%$ are living with HIV (UNAIDS, 2018).

The prevalence of HIV among PWID had been relatively low in Scotland since epidemics in Edinburgh and Dundee in the 1980s (Robertson et al., 1986; Urquhart et al., 1987). However in 2015, an outbreak was detected among PWID in Glasgow and has led to a ten-fold increase in HIV prevalence among this high-risk population in the city (McAuley et al., 2019). This is the largest HIV outbreak among PWID in the UK for 30 years, and has been shown to be associated with incarceration, homelessness, public injecting and a major increase in intravenous cocaine use (McAuley et al., 2019; Trayner et al., 2019). It is clear that there are multiple, complex, distal and proximal determinants of HIV risk.

Multi-levelled initiatives have been implemented to respond to the HIV outbreak in Glasgow, including increased provision of injecting equipment services and an enhanced model of HIV care involving outreach nursing and community prescribing of antiretroviral therapy. This has engaged more than $90 \%$ of the cohort in HIV treatment (Metcalfe et al., 2019). One approach that has not been provided for those at risk of injecting-related transmission in Glasgow is oral tenofovir disoproxil/emtrictibine as pre-exposure prophylaxis (PrEP), the use of a daily pill prior to exposure to HIV to minimise risk of infection (Brady, 2019). Randomised controlled trials (RCT) of people at risk of becoming infected sexually (particularly men who have sex with men) have demonstrated a significantly reduced risk of HIV 
acquisition among those prescribed PrEP (Grant et al., 2010; Fonner et al., 2016; McCormack et al., 2016). In 2017, Scotland was the first country in the UK to adopt a national HIV pre-exposure prophylaxis (PrEP) programme, as part of routine statefunded care to people who meet risk-based eligibility criteria (HPS, 2019). The service in Scotland involves provision of oral antiretroviral drugs to people at sexual risk of HIV acquisition. However, the approval of PrEP medicines in Scotland does not extend to individuals at high risk of HIV acquisition through injecting drug use (Scottish Medicines Consortium, 2017). Despite 2015 WHO guidelines recommending PrEP for all populations at substantial risk of HIV infection (WHO, 2015), so far there has only been one RCT with PWID (Choopanya et al., 2013). It showed a significant reduction in HIV incidence among recipients of PrEP. However, there were some potential confounding factors: increased patient engagement with Opiate Substitution Therapy (OST); increased use of clean injecting equipment; and reduction in sexual partners which may have also contributed to reduction in HIV incidence (Craig et al., 2013).

Research on the potential of PrEP from the perspective of Service Users has demonstrated high willingness among PWID to use PrEP, but this was contingent on: adequate levels of knowledge about PrEP; social motivation for taking medicines; personal motivation; and individual self-perceptions of HIV risk (Escudero et al.,2014; Stein et al., 2014; Shrestha et al., 2017). Studies that focus on Service Provider perspectives suggest that willingness to prescribe PrEP for PWID is contingent on their knowledge and experience of working with PrEP, alongside perceptions on the extent to which the target patient group will adhere, maintain, and engage in regular follow up to the treatment (Adams \& Balderson, 2016; Krakower et al., 2016; Edelman et al., 2017). None of these studies have been conducted in the 
context of an HIV outbreak among PWID, considering PrEP as a potential tool to control the spread of infection. Despite well established and extensive harm reduction services in Glasgow, the outbreak has persisted for more than 5 years. Glasgow thus represents a relatively unique setting to assess how PrEP might be introduced in combination with existing HIV prevention services to address the particularly high risk population PWID.

This study aimed to elicit the views and perspectives of PWID who may access the service (Service Users) and those who are likely to provide such a service (Service Providers). The research particularly focussed on determining how a PrEP service might be tailored to the population, socio-cultural context and service landscape, and what this means for PrEP delivery to PWID more generally. Specifically, our objectives were to understand: diversity in PrEP literacy and PrEP willingness amongst Service Users and Providers; the barriers and facilitators to implementation; and the barriers and facilitators to uptake and engagement by those at high risk of HIV.

\section{Methods}

\section{Approach}

A phenomenological qualitative approach (Groenewald, 2004) was adopted for this research, as It was vital to place the experiences and understandings of participants at the centre of data collection and analysis. Semi-structured interviews were conducted in order to prioritise space within data collection for participants to highlight the issues they found to be most important, but also to allow some steering of questions to address some specific issues. This allowed us to take an inductive approach to data collection, whilst still maintaining the ability to explore specific 
factors around service engagement and provision apparent from previous research on PrEP, HIV and PWID service engagement. Interviews took place in Glasgow between April and September 2018 with potential Service Providers and Service Users of PrEP. Recruitment was carried out until data saturation was reached (Fusch, 2015). For Service Providers, inclusion criteria were that they were professionally involved in a service that had direct contact with current PWID. They were purposively recruited to gain a range of both frontline and strategic staff from a variety of statutory and third sector organisations. Service Provider interviews took place at either places of work or quiet public spaces such as cafes, as determined by the participants.

For Service Users inclusion criteria were that they were able to give informed consent at time of recruitment and interview, were over 18 , were either currently injecting or had recently injected drugs (within the last 6 months), and lived in the City of Glasgow. Participants were recruited through two third sector non-NHS service settings (a homeless drop-in centre and a drug crisis centre) both situated in Glasgow. Staff members advised the researcher if particular individuals did not have capacity to take part due to physical or mental health distress. Otherwise potential participants were approached within the service and provided an information sheet and an opportunity to ask questions, and schedule a convenient time for interview. All interviews were conducted at the services the participants attended and within private rooms. Written informed consent was taken at the point of interview. Interviews lasted a maximum of an hour and Service Users were given a £25 shopping voucher as compensation for their time. In order to ensure that participants did not feel pressured to take part, it was re-iterated repeatedly that their inclusion in the study would not affect their engagement with the gatekeeper organisation 
through which they were recruited. In addition, they were reminded often that they could stop the interview at any time, and still be given the full voucher amount, regardless of time in interview.

Service Users were recruited purposively to gain a gender balanced sample and a diverse age range. Other demographic dimensions (e.g. sexuality, ethnicity, national origin etc.) were not used for the purposive sampling due to two factors. First, HIV PWID epidemiology in Scotland does not identify any significance related to sexuality, ethnicity or nationality (McAuley et al., 2019). Secondly, due to the limited opportunities for recruitment through gatekeepers, and limited time and resources available for the project, sampling along these dimensions would have been extremely challenging. A wider sampling framework may be more appropriate for follow up research (see discussion).

Service users were not recruited based on HIV status, nor were they asked whether they had HIV before taking part. It was decided that asking HIV status at the recruitment stage was unethical, and would increase chances of identification of individuals, and potential unwanted disclosure of HIV status. As participants were being recruited through gatekeeper organisations, it was also decided that doing this might force unwanted disclosure of participant's HIV status with those organisations. It was also decided that if HIV positive participants did decide to disclose their status independently during interview, they would not be excluded. These individuals were undoubtedly high risk by the very nature that they had HIV, and most likely acquired infection recently in the context of the outbreak situation, so their perspective on the role of PrEP was key. 
Ethical approval was gained from the Glasgow Caledonian University Ethics Committee for Nursing and Community Health.

Topic guides, information sheets, and consent forms were developed collaboratively with people with lived experience of substance use. This was done through a NonGovernmental Organisation (Scottish Drugs Forum) who facilitate people with lived experience of substance use contributing to Public Patient Involvement (PPI) in research projects. The organisation facilitated discussions and feedback on research tools above to ensure they were relevant and accessible to potential research participants. Interviews were semi-structured to allow flexibility in interview topics. For Service Providers, topic guides covered: knowledge and understanding of PrEP; predicted barriers and facilitators to a PrEP service; potential service design; how PrEP might fit into the current service landscape; and feasibility of introduction of PrEP. For Service Users, topic guides covered: sociodemographic context; exposure to injecting drugs and sexual risk; PrEP awareness and understanding; priority recipients of PrEP; barriers and facilitators to initiation and maintenance of PrEP; and how PrEP might affect substance use and service engagement.

Data were analysed using the "reflexive thematic analysis" approach, as defined by Clarke et al., (2015). Given our focus on generating inductive insights into the perspectives of those accessing and providing services it was important that the analysis primarily captured how participants understood the issues at stake. However, we also wanted to answer specific research questions, and apply some deductive coding. The reflexive thematic analysis approach allowed theoretical flexibility, and is suited to questions related to experiences, understandings and constructions of meaning (Clarke et al. 2015). No single a priori theoretical frame 
was used within the study. Where relevant we discuss these findings in relation to key theoretical constructs.

Analysis developed as follows. Initially researchers familiarised themselves with data, and began open coding on a sub set of five transcripts to capture inductively emergent themes, and give these codes. Once no sufficiently distinct themes were identified within this subset, codes were collated, with those of sufficient similarity combined. Simultaneously, deductive codes developed from research questions, which shaped interview topic guides, were applied to the same subset of transcripts. Again, sufficiently similar codes in these two preliminary sets were combined. At this stage these preliminary codes were reviewed by the broader research team and applied to a second subset of transcripts, and discrepancies and duplication lead to themes being adjusted through review by the research team. These "central" codes were sufficiently broad enough to cover all relevant data, but distinct from each other. Subsequently, these final agreed upon "central" codes were applied to the entire data set, so that all transcripts were coded with the same set of codes This was done using NVivo qualitative analysis software (Bazeley \& Jackson, 2013). The researcher (MS) applied the central codes and these were quality checked throughout the study by members of the research team. Once all data had been coded with central codes, each of these were sub-coded to multiple levels until data was organised at a sufficiently detailed level to allowed research aims to be addressed. Both Service User and Service Provider interviews were coded with the same list of codes.

It was assumed that the majority of participants would not have heard of PrEP prior to the interview. A scripted explanation was decided against, as different participants might have different levels of health literacy, but the researcher instead explained 
PrEP in as plain language as possible, describing it as a daily pill that could be taken to potentially prevent infection with HIV. As a starting point, this allowed participants to ask enough questions until the researcher was satisfied they had sufficient grasp on the concepts to be asked questions about it. The researcher was also careful to list potential side effects, so that participants had a balanced understanding of the benefits and risks of PrEP as a medication.

\section{Results}

In total, six themes related to PrEP were identified by researchers as being most important for this study: HIV and PrEP literacy; Willingness to use PrEP among PWID; HIV health promotion and potential lessons for PrEP; Implementation of PrEP services; and maintenance and adherence with PrEP. The themes explored here were not the only ones identified from study data, but those which were decided by the research team to be the most relevant to potential PrEP provision for PWID, barriers and facilitators to that provision, and indicative of areas amenable to change at a structural and individual level, and therefore most important to be published from this study.

\section{Sample}

11 interviews were undertaken with Service Providers encapsulating strategic and frontline staff in statutory and third sector organisations working directly with PWID. These comprised: a public health consultant; an HIV clinician; an HIV service manager; an HIV nurse; two third sector organisation managers; an outreach pharmacist; an outreach nurse; a pharmacist and two frontline substance use third sector organisation staff. 
21 interviews were carried out with Service Users ( $n=13$ men, $n=8$ women). (Table 1) Ages ranged from 21-61 years old, with a mean and median of 36 years. Five Service User participants self-reported that they were HIV positive, and three selfreported that they had $\mathrm{HCV}$, however this was not directly asked and others may have had HIV or HCV but chosen not to disclose. This is indicated in participant descriptions for service users: HIV positive; HIV negative; HIV unknown. All Service Users reported they were on Opiate Substitution Therapy (OST) at the time of interview. 13 were recruited from a drug crisis centre, and eight from the homelessness drop in third sector organisation. All participants have been given pseudonyms to protect their anonymity.

\begin{tabular}{|l|l|l|l|l|l|l|}
\hline \multirow{2}{*}{} & \multirow{2}{*}{} & N & Age & \multicolumn{4}{l|}{ BBV status (where known. Some participants did not disclose status) } \\
\cline { 3 - 7 } & & HIV positive & $\begin{array}{l}\text { HCV } \\
\text { negative }\end{array}$ & $\begin{array}{l}\text { HIV \& HCV } \\
\text { positive }\end{array}$ & HCV cleared \\
\hline Men & 13 & $21-61$ & 4 & 2 & 1 & 2 \\
\hline Women & 8 & $22-42$ & 1 & 1 & 1 & 5 \\
\hline
\end{tabular}

Table 1: sample details

\section{HIV and PrEP literacy}

Overall HIV knowledge among the Service User sample was variable. Most had knowledge of basic HIV health promotion messages, especially concerning needle sharing and blood to blood transmission. However, many appeared to have been under-served by HIV risk reducing initiatives and mainstream education concerning blood borne viruses: some still viewed HIV as a terminal illness; some displayed poor understanding on the importance of cleaning injecting equipment, and risks involved in reusing equipment; most displayed a general lack of knowledge about viral load and the concept that HIV can be suppressed; and most reported a lack of knowledge of HIV treatment. 
Many Service User participants displayed 'unrealistic optimism' (Shepperd et al., 2016), a classic human trait in which people show an "it will never happen to me" attitude. The fact that PWID were so underserved in relation to HIV literacy meant that in general, they did not pay attention to HIV, had not made efforts to seek information about and did not think HIV was relevant to their lives.

You just don't think it's [HIV] gonna happen to you. I caught Hep C plus Hep B. I was shattered. But HIV I've always thought I would never catch it. But just recently l've been putting myself at risk quite a lot. I was embarrassed going to the chemist... so I would find needles in the street... people like I say, people think that they won't catch it. And I know a lot of people last year who caught it and they can't believe that they're HIV positive, you know. (Steven, 38, HIV -ve)

Participants also reported that for most PWID, HIV lacked salience. Moreover, as well as being structurally underserved in relation to HIV education/HIV risk reduction initiatives, their apparent low HIV literacy and prioritisation was also related to the overwhelming prioritisation of their substance use, and more pertinent health concerns, for example, abscesses. This combined with continuing high levels of stigma around HIV meant that discussions and knowledge-sharing within the community about HIV were extremely rare. In addition, awareness of risk and HIV literacy did not necessarily translate into changing behaviours around injecting drugs for these participants. Steven (above) was typical of the Service User sample, aware of the risks but continuing to engage in risky behaviour. HIV literacy was therefore not a matter of 'knowing' or 'not knowing', but far more complex for this group, resulting from a range of distal and proximal influences. 
Only one Service User was aware of PrEP, through a friend who had been offered it as HIV prevention through MSM targeted services. Among Service Providers, all but two had heard of PrEP, and there was varying knowledge, with respect to: the specific drugs used for PrEP; its roll-out in Scotland through sexual health services; and requirements for PrEP to work, for example the importance of adherence to medication.

\section{Willingness to use PrEP among PWID.}

All Service Providers questioned why PrEP was not available to PWID in Glasgow. Service Users were overwhelmingly enthusiastic about the prospect of PrEP, once it was fully explained what PrEP was, how it might work, and what the effects would be including side effects. Particularly poignant were participants who self-reported being HIV positive, all of whom suggested they would have taken PrEP had it been available, and all of whom thought it might have prevented them becoming HIV positive. Perhaps more importantly, without prompting, a large majority of participants expressed particular interest in taking PrEP in the context of the HIV outbreak:

R: Do you know something that [PrEP] would be great, you know, because I think it's something that's real now it's, to me, it's important. It's, like I said before, I'm in the high risk group.

I: What do you mean when you say it's real now? Can you explain that a wee bit?

R: It's real because I know of so many people that's got [HIV] whereas before I knew virtually no one who had it. (George, 41, HIV -ve) 
In this context, the majority of the sample suggested they would use PrEP as a "safety net" in case of unexpected incidents. These participants conceptualised PrEP not as a way of offsetting routine risky behaviour but to mitigate accidental or nonroutine risky behaviour stemming from events like accidental needle stick injuries but also fluctuations in mental health, substance use and other sudden changes in circumstances. This was explained in the context that the majority would not wilfully put themselves at risk. Regardless of this, they would want to be on PrEP in case something happened. Importantly in these accounts, sexual transmission was not mentioned, demonstrating that this was a particularly ignored area of risk.

Taking [PrEP], aye, well I wouldn't share again, I dae get clean needles all the time... [but I would want it] just in case somebody swaps...like their bags [of heroin] about, and says, aye I've just pulled it out the wrapper and you can easily just take your eyes off it for two seconds... Anything at all... or even like a wee prick or something or like touching somebody in their pocket or something and they've got an uncapped needle... and you get stuck. (Andrew, 35, HIV -ve)

In sharp contrast to these results however, when discussing perspectives on whether the wider population of PWID would engage with PrEP, Service Users and Providers suggested that there were fundamental barriers to willingness to engage, these were multi-level but included clearly psychological issues such as lack of self-worth; connected lack of self-ascribed risk; but also wider structural issues such as being under served in relation to health literacy and the salience of HIV and PWID stigmas. These multi-levelled barriers were inter-dependant, all resulted in a lack of motivation to engage, and were all related to what participants perceived as the hierarchy of needs for PWID, which in most cases were framed by substance use. 
The difference in this group and our MSM population is health literacy and motivation, really it comes down to I think. So, you know, our sexual health services, are full of motivated MSM who are health literate to do research, who talk amongst each other, who are involved in activism and will come forward and bang on our doors for PrEP. And the fact is that this group of people have very different priorities in life. It's really difficult. (HIV consultant)

The complexities of PWID daily lived experience, including the potentially overwhelming drives around substance use, meant that PWID tended not to prioritise actions to improve health, particularly pre-emptive actions such as the use of PrEP. Many did not engage with health providers even when they had an issue causing severe physical degradation requiring immediate attention such as an abscess.

They're needing that drug there and then...now they're on the streets, you can't see a way out anyway, now they probably don't want to be here, so they're probably not even caring, you know what I mean, and even probably ones who... do know that they're maybe taking a risk, but maybe think it's not going to happen to me, or maybe at that point they don't care either. (Stacy, 35, HIV -ve)

While Stacy speaks of 'others', further discussion indicated that she had found herself in this position, and experienced the lack of self-worth and extremely low aspirational drive that was reported as a significant barrier to engagement among PWID. This was reported by participants as even more likely when PWID were at more extreme ends of substance use. When asked what "extreme substance use" manifested as, participants suggested:: consuming large quantities of drugs; engaging in poly-drug use; sleeping on the streets; sharing needles frequently; 
involvement in high levels of illegal activity; or suffering severe mental health problems like psychosis.

When both sets of participants were asked about what could be done to overcome these complex barriers to engagement, both referred to the concept that PWID can be engaged with during times of stability, or "windows of opportunity". One example of this was prison:

[They're more likely to engage because] they're more sober in the jail and they think about it a lot more, when they're outside they're mad with it. The only thing they're thinking of is... the next bit of drugs. Whereas... you've got a lot more access to healthcare in the jail and they offer you it as soon as you come in you go into the reception. The majority of people say 'yes!'. And then they just follow you up and they do it. (Ben, 43, HIV +ve)

Although he was talking about others here, it was clear during the interview that Ben's journey had also included significant engagement with healthcare within prison. Ben talks about "access" in prison being an important factor, but also the space to "think about it a lot more". The moments in PWID's lived experiences where they were able to consider their situations and have will to improve them took place outside prison as well, for example during residential rehabilitation, upon gaining housing, or in intense engagement with another organisation like a third sector organisation.

Participants consistently contrasted two mind-sets in their life course and substance use, one of "desperation" and one of sobriety or "cleanness". These mind-sets were often thought of as oppositional. Rosanna here recounts a time when "clean", that she considered the decisions she was making when she was using, including 
sharing injecting equipment with people who she suspected might be HIV positive. She suggests that she was not sure she would reject the option of sharing equipment even if she knew the other person was HIV positive. To her this illustrates how different her decision making processes and priorities are when she is using:

I actually thought to myself, like, I wonder if I would have still used it if she said she was HIV, do you know what I mean. Like, you're just fighting back in your own head, when you're clean. Because when you're for the drugs, you just don't, I don't know, nothing seems that important in your head apart from where you're getting your next drugs fae. (Rosanna, 30, HIV -ve)

These two mind-sets did not relate to changing awareness or perceptions of HIV risk, as many knew of the risks when engaging in behaviour such as needle sharing. But instead mind-sets were related to the extent to which risk evaluation affected behaviours.

In addition to directly addressing the determinants of drug use (e.g. social and economic inequality, stigma and marginalisation), and improved approaches to health and HIV literacy, some of our participants also highlighted the value of intervening at the individual level. For example, "windows of opportunity", where Service Users were not engaged in heavy drug use, were points of stability in which they reflected on the varied risks of their behaviour and environment. Some participants also felt that these points of stability could be used to learn health protecting mechanisms that could be carried through to periods of more intense substance use. During these times, they could build momentum to develop more positive health behaviours and decision making. Therefore they suggested these periods represented opportunities to engage with HIV PrEP, in ways that could 
continue into times when they would not be receptive to changing behaviour or protecting themselves.

\section{HIV Health Promotion and potential lessons for PrEP Promotion}

Our analysis points to the centrality of addressing the upstream determinants of drug use, health literacy and HIV literacy. However, given the immediacy of the HIV outbreak in Glasgow it was also important to address ways to compensate for the historical lack of effective HIV health promotion by providing community and individual-level HIV health promotion. Both groups of participants thought health promotion should be accurate, consistent and specific rather than generalised. This was framed in terms of lessons learned around safe injecting and HIV health promotion:

So there's a tone of, do you know, if you share needles you might catch HIV. But actually saying, do you know, this is a map of Glasgow so this is where all the known cases of HIV are. So it's in the street that you...your friend, you're in, that's where the cases are. So this is where it started and so the red colours like 2017 and then 2018 this is what it's like. This is how real it is. (Drop in manager)

This specificity was particularly emphasised as important by Service Users, who suggested PrEP related health promotion needed to be built on specific local understandings, so that PWID would consider concepts like personal and environmental risk to be pertinent to their daily lived experience rather than abstracted concepts that did not connect to them as individuals. Both samples reported that services had to do better in engaging in detail with the realities of the PWID experience in Glasgow. This was identified as absolutely vital in developing 
effective health messages and encouraging and promoting PrEP use. However, the most important contextual detail identified by both samples was the ongoing HIV outbreak. Using the outbreak as a lever in health promotion elevated it over generic messages which were perceived as being less relevant.

I: Do you feel that people would pay attention to [health promotion]?

R: Aye, definitely. With the way it is and that I think they would.

I: So, when you're saying the way it is you mean...?

R: The way it is with HIV, people getting diagnosed every day with it. I think they would pay attention. If it's to stop you fae getting it they're gonnae take it, aren't they? I would take it. Cause a lot of people out there that's got it and they don't tell anybody. You know what I mean? So, people will take it 'cause they know people lie about no having HIV. (Jamie, 36, HIV +ve)

When Service Users were asked what their preferred method of delivery was for promotion of PrEP, the overwhelming response was via peers highlighting the importance of community, social and peer networks as key determinants of health in these populations. A repeated account was identified about the importance of empathy and shared experience to quickly build bonds of trust that facilitated effective communication of health messages, especially in terms of the immediacy, severity and salience of the message.

You kind of pay attention I think. If somebody sits and tells you aboot drugs and aw that and has never used drugs... well, you dae listen but I think in a way you listen better if it's somebody that's been through things. (Mark, 28, $H I V-v e)$ 
Many participants suggested they would be more likely to pay attention to someone discussing PrEP if they had caught HIV as a person who injected drugs. The HIV positive participants had been shocked and surprised by their diagnoses, and had either not considered the risk at all, or not considered themselves to be at risk. Messages about immediacy of the risk of HIV were more impactful coming from people that had lived through that context and experience.

Other addicts, people that's been through it. People that are on the treatment, and aren't embarrassed. People that have caught the HIV, and aren't ashamed about it, are open about it, they can talk about it. It's experience. You cannae buy experience. People that's experienced, getting the virus, and didnae have the chance to protect themselves. Because they've been through it, they've experienced it, they know what it's like. Instead of somebody that's just come out of college, they don't know what it's like... people that have been through, slept on the streets, begged, used needles, used other people's needles. (Rosanna, 30, HIV -ve)

Several participants suggested that there had to be nuanced understanding in deployment of peers. Rather than just anyone with lived experience, for PrEP a more impactful message would come from those who are HIV positive due to the same contexts and behaviours as the targets of health promotion.

\section{Implementation of PrEP services}

Another way of addressing the systematic disadvantages and vulnerabilities often experienced by PWID communities is to intervene at the level of service provision to address immediate needs and the consequences rather than the determinants of disadvantage/being under-served. 
The unprompted and overwhelming response from both groups when questioned about implementation of a PrEP service was that it should be delivered through community pharmacies alongside current OST programmes. Pharmacies were suggested to be key to reaching the largest number of PWID as possible.

See most people are on methadone or suboxone so they've got to go to a pharmacy every day or even if they're not on them they've got to go to a pharmacy to collect needles. So part and parcel of getting your methadone, your suboxone, or your needles, is by taking a PrEP pill. I think that would be a great way of getting it into the community. (Gareth, 40, HIV unknown)

Gareth highlights that everyday attendance aligns well with the need to take one PrEP pill per day, which would encourage adherence. Many of the other Service Users suggested that collecting methadone was the only structural regularity in their daily lived experience, a foundation of their daily plans (one referring to it as his "protocol"), especially for those who were at the more extreme end of drug use.

The other reason that pharmacies were identified as an ideal site of provision was that generally PWID were reported to have positive and trusting relationships with pharmacists, and participants emphasised how their first-hand experience repeatedly highlighted the vital importance of relationships of trust for PWID engagement.

I've seen me going back to the chemist, l've been going to the same guy for years. And this guy, oh, he's a total professional, man. So that's why, he's gonna make sure that you've had it, he's brilliant. (Alec, 43, HIV +ve)

Pharmacists represented an empathetic point of engagement, with whom many Service Users had developed a relationship and rapport with over a long period. 
Service User participants reported that they were confident in being treated with respect and empathy for the most part, and that they could engage in a confidential and discreet way.

Well a lot of people... don't trust you very easily, it can take a wee while to build up relationships with people especially sort of homeless people, drug users that have had a hard time or treated badly by services in general and still are ... It takes a little while to build up trust and confidence and give them a little bit of information sort of slowly kind of drip, drip effect. Letting them know that you do care, that you're thinking about them, that you remember them, you'll sort of come back to them again and eventually they do, you know, sometimes it takes a while but they will eventually engage and trust you. (Outreach nurse)

Long term, regular, close and supportive contact, characterised here as the "drip drip" approach, was vital. Participants were keen that a new service, like provision of PrEP, would be developed with these core values.

Not all participants were supportive of the pharmacy model, and several Service Provider participants highlighted the following issues with deploying PrEP through pharmacies: the pragmatic complexities of prescribing PrEP and monitoring ongoing usage; oversight and cost issues; and the extent to which pharmacies would be able to engage with the most isolated, least resourced and most vulnerable PWID.

There's people who don't seem to be engaging in OST, the risks increase... The likelihood of them engaging in medical intervention decrease at the same time... somebody like that goes into freefall until they are either put in prison or they land at...so far down that they're left with nae options... So during that 
freefall period getting people to engage is... gonna be severely problematic. (Prison link worker)

The extent to which this approach would be able to engage with the most vulnerable, in "freefall", was the main criticism of an approach that attempted to build on preexisting pathways. For these Service Provider participants, within the current engagement context many PWID are neglected. As such any those same PWID would be neglected in a PrEP delivery approach that built on the current pharmacy services.

\section{Maintenance and adherence with PrEP}

Another area of concern which was identified in interviews was the extent to which PWID would be able to adhere to the strict conditions required for effective PrEP use. At the time of writing, PrEP comes in the form of daily tablets, and if doses are missed, it compromises the efficacy of the drug. The majority view from both sets of participants was that in principle, adherence would not be an issue. Many participants cited their own experiences or examples of successful adherence to complex medical regimens such as anti-retrovirals and anti-psychotic medication. Some Service Users suggested they were currently taking a range of medication and adding another to the list would not be a substantial issue.

One pill a day would be brand new [really good]. Easy-peasy man. I already take three pills a day as it is because l've got a paracetamol a day and that for my headaches, and other stuff. Another [pill] for my collection. (Andrew, 35, HIV -ve)

However, a significant minority from each group suggested that "chaotic lives" of PWID could be problematic for maintenance: 
The group we work with are... extremely vulnerable ... largely chaotic ... if it was a choice between go and get your medication or I'm rattling and I need to get money and I need to go and score ... well the medications getting left ...whatever happens after the using can be more dependent on who the person is with ... and how they're going to use their next bag, rather than I need to go to the other side of the city to get my medication. (Outreach Worker)

Potential unpredictability of the daily existence of PWID, similarly to issues around will to use PrEP, might result in people forgetting medication, losing it, or otherwise not prioritising taking it on a daily basis.

An outreach pharmacist identified that they thought the main issue with PrEP adherence would be a lack of immediate feedback that was quantifiable and perceptibly beneficial physically, in contrast to a reactive medicine like antihypertensives:

We started quite a lot of people on anti-hypertensives. Blood pressure, and the ones that tend to take it regularly are the ones who notice that they're feeling unwell when they don't take it. I don't think we would get that in terms of PrEP. I don't think there's any...I don't think there's any strong telling reason for the person to take it there and then every day... for a risk that they can't quantify for themselves, you know. (Outreach pharmacist)

In addition, even for medication where they should be able to "quantify" the benefits, some Service Users admitted historical issues with adherence, due to complexity with schedules, and the lack of motivation and health literacy, as previously discussed, during periods of high-level substance use. 
They gave me it for my leg, antibiotics four times a day and aw that and I couldnae take them. If it's no in my heid, in my heid's... when I'm on the street, see when l'm oot there using all that's in my heid it's just drugs and aw that and money, how to get money and aw that. (Mark, 28, HIV -ve)

\section{Discussion}

Against a backdrop of multiple disadvantages and a history of being systematically underserved this research demonstrateda range of compensatory approaches that target the proximal determinants of HIV transmission amongst PWID including the provision of PrEP and related services. In our discussion we take a socio-ecological approach to results to ensure that findings related to individual behaviours and motivations are appropriately contextualised within broader socio-ecological factors.

Our findings show a willingness for PrEP among PWID and potential Service Providers, especially as a potential tool to lessen risk of HIV acquisition within the context of an ongoing HIV outbreak. Service Users and Service Providers suggested that PrEP would be useful and questioned why it had not yet been provided to this population, especially in the context of an ongoing HIV outbreak. Whilst study participants were enthusiastic about PrEP, they also identified potential barriers to engagement: lack of health literacy and motivation; lack of self-ascribed risk; and related issues connected to the overwhelming unpredictability and uncertainty of injecting drug use. Participants suggested PrEP should be promoted to PWID in a manner that is relevant to their daily lived experiences within the local context. They suggested PrEP should be provided in a way that aligns with existing services in regular contact with those at risk such as pharmacies, and should take advantage of the resources represented by peers to communicate compellingly. Also participants 
suggested that in order for PrEP provision to be effective, promotion and engagement must take into account "windows of opportunity" among Service Users; defined as times where they are likely to be more receptive to health related, particularly preventative, messages.

This data, like other studies, illustrates the potential complexity of encouraging behaviour change and service engagement for PWID (Biello et al., 2018). The fact that an ongoing outbreak is continuing despite apparently widespread OST and needle and syringe provision service availability suggests that provision alone will not be enough to engage PWID with a future PrEP service. Instead the current research suggests that multi-level approaches dealing with barriers to engagement across individual and health service systems simultaneously have improved chances of success. An example of this in Glasgow has been the HIV clinical outreach approach that has been developed to ensure those with HIV are on therapy. Glasgow has now achieved very high coverage of anti-retroviral therapy for those diagnosed with HIV since the beginning of the outbreak, with $86 \%$ of that cohort achieving viral suppression by June 2019 (Metcalfe et al., 2019). However, levels of engagement and retention in HIV care were low in the early years and clinical services adapted to address this, including developing services with lower thresholds to entry and retention at the same time as funding more intense street based outreach and engagement efforts (Metcalfe et al.,2019). Whilst this is a much more resource intensive approach, it is also proof of concept that high engagement, retention and adherence rates can be developed in this specific population.

The accounts of those with lived experience and to a lesser extend service providers are focussed on individualist behaviours and motivations that act either as barriers or facilitators to PrEP. Addressing these alone are unlikely to bring about change in the 
distal factors that generate and sustain the conditions for problem drug use including HIV infection. Viewed through the lens of the social ecological model (Kilanowski, 2017), the accounts of service providers and those with lived experience acknowledged the importance of engaging with credible peers as part of the response in addition to developing a network of street based services. This represents a paradigm shift whereby greater emphasis in placed on upstream action including organisations that monitor and promote change at the local level and interpersonal connections that foster collective action (Golden et al 2015).

Given the lack of prioritisation of HIV, and potentially a preventative medication such as PrEP, the sample reported mixed perspectives on whether a new PrEP service would be able to pull in those who would most benefit among PWID populations. As such, participants suggested that PrEP services should build on relationships with existing services such as pharmacists, however with caveats that this cannot be the only delivery of PrEP in order that those who do not currently engage with pharmacies also have pathways to PrEP provision so as to include non-engagers with pharmacies. In addition, the approach discussed above of intense outreach combined with lowered entry and retention thresholds for services could improve engagement through a pharmacy based approach. Another issue with the pharmacy approach is that it assumes a similar system to what exists in Scotland, which is not the case in many countries. Potentially other services with good records of engagement that are perceived positively by PWID would also work, but more exploration is required. Aligning services to encourage attachment, whether this be through prescribing PrEP alongside current engagement, or improving flexibility in opportunities for PWID to attain PrEP should be prioritised. It is evident from this research that individual experiences of substance use and service engagement are 
highly varied. Bazzi et al., (2019) in their systematic review of HIV treatment adherence by PWID in North America to inform future PrEP services recommend a nuanced and in-depth understanding of individual drug use patterns and socioenvironmental contexts as a pre-requisite to effective PrEP provision.

In addition at a service level, the current research indicates that services should endeavour to take into account the fluctuations of PWID's capacities to engage, and look to capitalise on identified "moments of clarity". In practical terms this means flexibility and allowances for potentially inconsistent engagement. Hershow et al. (2019), promote using a foundation of patient self-efficacy as a starting point for design of health service engagement interventions. If interventions are designed with this as a priority, it might lead to a useful reservoir of knowledge within PWID groups that can be transferred horizontally. This in turn could take advantage of those times where individuals are receptive to health messages from their peers and others, rather than the current approach of regular scheduled appointments that do not necessarily fit with the fluctuations in self-efficacy in PWID (Kadden et al., 2011). In addition, developing strategies to increase overall self-efficacy could be an effective approach to widen these "moments of clarity", facilitating greater opportunities to affect engagement and behaviour change. This is not a problem unique to PrEP engagement, but the extent to which this is considered might determine success or failure for PrEP provision to this population. Until long-acting PrEP (for example subdermal applications (Johnson et al., 2019)) is available, the success of PrEP for this population will rely on approaches that motivate users to engage regularly.

Given the complexities apparent in PWID population around self-efficacy, engagement, lack of prioritisation of HIV, wide variations in self-ascribed risk, and other dimensions, PrEP provision should therefore be predicated on a wide and 
diverse range of referral pathways, with low thresholds for assessment and recruitment, again in contrast to PrEP for MSM in Scotland, which is currently only available through sexual health clinics (Frankis et al., 2019). As suggested by a socio-ecological approach, policy, community, and organisational level interventions can be complimented by individualistic interventions. This study identified factors amenable to change at an individual level: encouraging engagement and health literacy around HIV is a priority, as is developing approaches that empower PWID to address the risks in their daily lived experiences. This research indicates that this should include: improving HIV literacy, particularly in terms of sexual transmission and vulnerability; and developing mechanisms to support more accurate selfascribed risk formulations. Arguably the most important aspect of this for Glasgow is to develop community-based specific awareness of the HIV outbreak. Developing specific localised knowledge that PWID can access within their daily lived experiences about HIV and the outbreak may be more effective in encouraging engagement than generic messages about avoiding needle sharing. This specific threat of increased chance of infection requires a different approach to that used with MSM, where PrEP is promoted on the basis of increased individual behavioural risks rather than environmental risks (Biello, 2018 ${ }^{1}$ ). The HIV outbreak can be used as a lever with which the impact of health promotion messages can be increased. Connected to this were results suggesting that peers who have had the same experiences as their target audience should carry out health promotion and that local detailed context is vital for applicability of health promotion messages. In addition, peer based promotion could take a "hierarchy of credibility" (Becker, 1967) approach where peers are deployed on the basis of the nuance of their experiences. Peers with differing specific experiences might be more effective in delivering different 
messages to different sub-populations of PWID. In conclusion a fundamental challenge is how to work towards changes in health and social care at the local level. Our research focussed of homeless people and the service staff who provided practical and emotional support to them. Service providers were acutely aware of the social and economic status of those with lived experience and their struggles with every day life. Disenfranchised and marginalised from society it is difficult to see how the voices of those who are homeless and in poverty will be driving force for change. However this might be possible if services and communities (including peer workers) work together to change the service landscape in localities where there is greatest need (Golden et al 2015). An example might be PreP delivered as part of a harm reduction programme that includes access to larger range of services including health and social care (Coleman and McLean 2016). This study has several limitations. Recruitment into the study happened in only two sites, due to issues with access to PWID (increased complexity in recruiting from NHS sites), and the requirement of workers with pre-existing relationships to act as gatekeepers. This meant a potentially limited diversity of PWID participants. However we did manage to recruit a sample with significant experience of homelessness and intense drug use, as well as individuals with HIV, a broad range of ages and a balance of men and women. However, with greater time and resources, a more targeted approach to recruitment could have meant sample stratification on the basis of specific patterns of drug use (e.g. frequency or substance type) in order to understand if this leads to a variety in perspectives on PrEP. Ideally it would also have been preferable to address other demographic dimensions (e.g. ethnicity, national origin, sexuality) to understand if this had any bearing on perspectives on PrEP and other themes. This should be sought in future studies. In addition, due to reliance on gatekeepers for 
recruitment strategies, some selection bias may have occurred outwith the control of the researcher towards participants who were deemed "suitable". Regarding recruitment sites, one was a residential rehab facility, in which many of the participants were aiming to discontinue injecting drug use, and therefore found it difficult to talk about perspectives on their own potential use of PrEP. In addition, this study was prospective. None of the participants had taken PrEP and therefore could not speak of lived experiences of PrEP treatment and associated barriers and facilitators, so much of the results are hypothetical, and based on analogous engagement experiences.

\section{What the study adds to existing evidence}

PrEP is a potential response to an HIV outbreak among PWID, but only if the message of promotion, and pathways to engagement, have high levels of salience to the target population. This means localised geographical messages concerning outbreak areas, specific messages that resonate with the lived experiences of PWID and low thresholds for engagement that consider the ongoing complexities of PWID within their own localised context.

\section{Conclusions}

This study provides unique insight into perspectives on PrEP for PWID from both potential users and potential providers in the context of and as a response to an HIV outbreak in order to inform future provisions of PrEP for this population. To the best of the authors' knowledge it is the first to do so. Service Provider and Service User participants have demonstrated enthusiastic willingness to engage, with HIV infected Service Users even suggesting that they could have avoided infection had PrEP been available. In our discussion we suggest that PrEP on its own may have an 
impact on the HIV outbreak, but without a wider interrogation of broader social and economic inequalities that lead to harms suffered by PWID, that impact may be limited. Indeed, recommendations that do not take this context into account may result in further widening of inequalities to the detriment of PWID who might benefit from PrEP provision. These contextual details must shape PrEP provision, otherwise engagement will meet substantial barriers. It will not be enough to adapt current provision to this population, as there are too many contrasts in engagement as shown by this paper. The perspectives, experiences and voices of PWID should be at the centre of any innovation or intervention to supply PrEP to this population. Glasgow has the benefit of being able to develop a PrEP service that can learn from the strengths and weaknesses apparent in current widespread engagement attempts in response to the ongoing HIV outbreak. Adapting clinical services, such as PrEP delivery, to improve accessibility and support adherence, can increase engagement and retention in care. This study has already informed a pilot service in Glasgow delivering PrEP to PWID who are at risk of HIV acquisition via sex, which will be expanded in future. Future research must build on the clinical evidence for efficacy of PrEP for PWID, but in the meantime pilot services should be developed which can meet the needs of PWID under current national recommendations. Given the wide range of PrEP services available for other populations at risk of $\mathrm{HIV}$, and the ongoing HIV outbreak among PWID in Glasgow, delivery of PrEP to PWID has become a clinical, social and moral imperative for health services.

\section{References}

Bak, M., van Dam, A., \& Janssens, R. (2018). Awareness and Acceptability of PreExposure Prophylaxis (PrEP) Among Men Who Have Sex with Men in 
Kazakhstan: A Mixed Methods Study. Central Asian Journal of Medical Sciences, 4(2), 102-115.

Biello, K. B., Mimiaga, M. J., Santostefano, C. M., Novak, D. S., \& Mayer, K. H. (2018). MSM at highest risk for HIV acquisition express greatest interest and preference for injectable antiretroviral PrEP compared to daily, oral medication. AIDS and Behavior, 22(4), 1158-1164.

Biello, K. B., Bazzi, A. R., Mimiaga, M. J., Biancarelli, D. L., Edeza, A., Salhaney, P., Childs, E., \& Drainoni, M. L. (2018). Perspectives on HIV pre-exposure prophylaxis (PrEP) utilization and related intervention needs among people who inject drugs. Harm Reduction Journal, 15(1), 1-12.

Brady, M., Rodger, A., Asboe, D., Cambiano, V., Clutterbuck, D., Desai, M., Field, N., Harbottle, J., Jamal, Z., \& McCormack, S. (2019). BHIVA/BASHH guidelines on the use of HIV pre-exposure prophylaxis (PrEP) 2018. HIV Medicine, 20(S2), S2-S80.

Clarke, V., Braun, V., \& Hayfield, N. (2015). Thematic analysis. Qualitative Psychology: A Practical Guide to Research Methods, 222-248.

Choopanya, K., Martin, M., Suntharasamai, P., Sangkum, U., Mock, P. A., Leethochawalit, M., Chiamwongpaet, S., Kitisin, P., Natrujirote, P., \& Kittimunkong, S. (2013). Antiretroviral prophylaxis for HIV infection in injecting drug users in Bangkok, Thailand (the Bangkok Tenofovir Study): a randomised, double-blind, placebo-controlled phase 3 trial. The Lancet, 381(9883), 20832090. 
Craig, A. P., Gray, R., Jansson, J., \& Wilson, D. P. (2013). HIV antiretroviral prophylaxis for injecting drug users. The Lancet, 382(9895), 854-855.

Coleman, R. L., \& McLean, S. (2016). Commentary: the value of PrEP for people who inject drugs. Journal of the International AIDS Society, 19, 21112.

Degenhardt, L., Mathers, B., Vickerman, P., Rhodes, T., Latkin, C., \& Hickman, M. (2010). Prevention of HIV infection for people who inject drugs: why individual, structural, and combination approaches are needed. The Lancet, 376(9737), 285-301.

Edelman, E. J., Moore, B. A., Calabrese, S. K., Berkenblit, G., Cunningham, C., Patel, V., Phillips, K., Tetrault, J. M., Shah, M., \& Fiellin, D. A. (2017). Primary care physicians' willingness to prescribe HIV pre-exposure prophylaxis for people who inject drugs. AIDS and Behavior, 21(4), 1025-1033.

El-Bassel, N., Shaw, S. A., Dasgupta, A., \& Strathdee, S. A. (2014). Drug use as a driver of HIV risks: re-emerging and emerging issues. Current Opinion in HIV and AIDS, 9(2), 150.

Escudero, D. J., Kerr, T., Wood, E., Nguyen, P., Lurie, M. N., Sued, O., \& Marshall, B. D. L. (2015). Acceptability of HIV Pre-exposure Prophylaxis (P r EP) Among People Who Inject Drugs (PWID) in a Canadian Setting. AIDS and Behavior, 19(5), 752-757.

Felsher, M., Szep, Z., Krakower, D., Martinez-Donate, A., Tran, N., \& Roth, A. M. (2018). "I Don't Need PrEP Right Now": A Qualitative Exploration of the Barriers 
to PrEP Care Engagement Through the Application of the Health Belief Model. AIDS Education and Prevention, 30(5), 369-381.

Fonner, V. A., Dalglish, S. L., Kennedy, C. E., Baggaley, R., O'reilly, K. R., Koechlin, F. M., Rodolph, M., Hodges-Mameletzis, I., \& Grant, R. M. (2016). Effectiveness and safety of oral HIV preexposure prophylaxis for all populations. AIDS (London, England), 30(12), 1973.

Fusch, P. I., \& Ness, L. R. (2015). Are we there yet? Data saturation in qualitative research. The Qualitative Report, 20(9), 1408.

Grant, R. M., Lama, J. R., Anderson, P. L., McMahan, V., Liu, A. Y., Vargas, L., Goicochea, P., Casapía, M., Guanira-Carranza, J. V., \& Ramirez-Cardich, M. E. (2010). Preexposure chemoprophylaxis for HIV prevention in men who have sex with men. New England Journal of Medicine, 363(27), 2587-2599.

Groenewald, T. (2004). A phenomenological research design illustrated. International journal of qualitative methods, 3(1), 42-55.

Golden, S, McLeroy K, Green L, J Earp, L Lieberman, (2015). Upending the Social Ecological Model to Guide Health Promotion Efforts Toward Policy and Environmental Change. Health Education \& Behaviour, Vol. 42(1S) 8S-14S.

Health Protection Scotland [HPS] (2019) Implementation of HIV PrEP in Scotland: First Year Report. Retrieved from https://www.hps.scot.nhs.uk/web-resourcescontainer/implementation-of-hiv-prep-in-scotland-first-year-report

Hershow, R. B., Gonzalez, M., Costenbader, E., Zule, W., Golin, C., \& BrinkleyRubinstein, L. (2019). Medical Providers and Harm Reduction Views on Pre- 
Exposure Prophylaxis for HIV Prevention Among People Who Inject Drugs. AIDS Education and Prevention, 31(4), 363-379.

Hope, V. D., Harris, R. J., De Angelis, D., Croxford, S., Marongiu, A., Parry, J. V, \& Ncube, F. (2014). Two decades of successes and failures in controlling the transmission of HIV through injecting drug use in England and Wales, 1990 to 2011. Euro Surveillance, 19(14), 15-26.

Janz, N. K., \& Becker, M. H. (1984). The health belief model: A decade later. Health Education Quarterly, 11(1), 1-47.

Johnson, L. M., Krovi, S. A., Li, L., Girouard, N., Demkovich, Z. R., Myers, D., Creelman, B., \& van der Straten, A. (2019). Characterization of a reservoir-style implant for sustained release of tenofovir alafenamide (TAF) for HIV preexposure prophylaxis (PrEP). Pharmaceutics, 11(7), 315.

Kadden, R. M., \& Litt, M. D. (2011). The role of self-efficacy in the treatment of substance use disorders. Addictive Behaviors, 36(12), 1120-1126.

Kilanowski, J.F. (2017) Breadth of the Socio-ecological Model, Journal of Agromedicine, 22:4, 295-297.

Krakower, D. S., Beekmann, S. E., Polgreen, P. M., \& Mayer, K. H. (2016). Diffusion of newer HIV prevention innovations: variable practices of frontline infectious diseases physicians. Clinical Infectious Diseases, 62(1), 99-105.

McAuley, A., Palmateer, N. E., Goldberg, D. J., Trayner, K. M. A., Shepherd, S. J., Gunson, R. N., Metcalfe, R., Milosevic, C., Taylor, A., \& Munro, A. (2019). Reemergence of HIV related to injecting drug use despite a comprehensive harm 
reduction environment: a cross-sectional analysis. The Lancet HIV, 6(5), e315e324.

McCormack, S., Dunn, D. T., Desai, M., Dolling, D. I., Gafos, M., Gilson, R., Sullivan, A. K., Clarke, A., Reeves, I., \& Schembri, G. (2016). Pre-exposure prophylaxis to prevent the acquisition of HIV-1 infection (PROUD): effectiveness results from the pilot phase of a pragmatic open-label randomised trial. The Lancet, 387(10013), 53-60.

Metcalfe R., Ragonnet-Cronin M., Bradley-Stewart A., McAuley A., Stubbs H., R. T. (n.d.). From hospital to community - redesigning the HIV clinical service model to respond to an outbreak of HIV among people who inject drugs.

Nyblade, L., Stangl, A., Weiss, E., \& Ashburn, K. (2009). Combating HIV stigma in health care settings: what works? Journal of the International AIDS Society, 12(1), 15.

Page, M., \& Nicholls, J. (2018). PrEP: pre-exposure prophylaxis for HIV prevention. Trends in Urology \& Men's Health, 9(4), 22-24.

Scottish Medicines Consortium. (2017). Emtricitabine/tenofovir disoproxil (Truvada®) is accepted for use within NHS Scotland. Retrieved 19/2/2020 from: https://www.scottishmedicines.org.uk/media/1620/emtricitabine tenofovir disopr oxil truvada final march 2017 for website.pdf

Shrestha, R., Altice, F. L., Huedo-Medina, T. B., Karki, P., \& Copenhaver, M. (2017). Willingness to use pre-exposure prophylaxis (PrEP): an empirical test of the 
information-motivation-behavioral skills (IMB) model among high-risk drug users in treatment. AIDS and Behavior, 21(5), 1299-1308.

Stein, M., Thurmond, P., \& Bailey, G. (2014). Willingness to use HIV pre-exposure prophylaxis among opiate users. AIDS and Behavior, 18(9), 1694-1700.

Thigpen, M. C., Kebaabetswe, P. M., Paxton, L. A., Smith, D. K., Rose, C. E., Segolodi, T. M., Henderson, F. L., Pathak, S. R., Soud, F. A., \& Chillag, K. L. (2012). Antiretroviral preexposure prophylaxis for heterosexual HIV transmission in Botswana. New England Journal of Medicine, 367(5), 423-434.

Trayner, K. M. A., McAuley, A., Palmateer, N. E., Goldberg, D. J., Shepherd, S. J., Gunson, R. N., Tweed, E. J., Priyadarshi, S., Milosevic, C., \& Hutchinson, S. J. (2019). Increased risk of HIV and other drug-related harms associated with injecting in public places among people who inject drugs in Scotland: a national bio-behavioural survey. The Lancet, 394, S91.

Organization, W. H. (2016). Guideline on when to start antiretroviral therapy and on pre-exposure prophylaxis for HIV. 2015.

Robertson, J. R., Bucknall, A. B., Welsby, P. D., Roberts, J. J., Inglis, J. M., Peutherer, J. F., \& Brettle, R. P. (1986). Epidemic of AIDS related virus (HTLVIII/LAV) infection among intravenous drug abusers. Br Med J (Clin Res Ed), 292(6519), 527-529.

Shepperd, J. A., Waters, E. A., Weinstein, N. D., \& Klein, W. M. P. (2015). A primer on unrealistic optimism. Current Directions in Psychological Science, 24(3), 232-237. 
UNAIDS (2018). Miles-to-go: closing the gaps, breaking barriers, righting injustices:

Global AIDS update 2018. Geneva; 2018.

Funding sources: This work was funded by the NHS Greater Glasgow and Clyde 